The Astrophysical Journal Supplement Series, 90:541-546, 1994 February

(C) 1994. The American Astronomical Society. All rights reserved. Printed in U.S.A.

\title{
JUPITER RADIO BURSTS AND PARTICLE ACCELERATION
}

\author{
MiChaEL D. DESCH \\ NASA/Goddard Space Flight Center, Greenbelt, MD, 20771 \\ Received 1993 February 25; accepted 1993 June 9
}

\begin{abstract}
Particle acceleration processes are important in understanding many of the Jovian radio and plasma wave emissions. However, except for the high-energy electrons that generate synchrotron emission following inward diffusion from the outer magnetosphere, acceleration processes in Jupiter's magnetosphere and between Jupiter and Io are poorly understood. We discuss very recent observations from the Ulysses spacecraft of two new Jovian radio and plasma wave emissions in which particle acceleration processes are important and have been addressed directly by complementary investigations. First, radio bursts known as quasi-periodic bursts have been observed in close association with a population of highly energetic electrons. Second, a population of much lower energy ( $\mathrm{keV}$ range) electrons on auroral field lines can be shown to be responsible for the first observation of a Jovian plasma wave emission known as auroral hiss.
\end{abstract}

Subject headings: acceleration of particles - planets and satellites: individual (Jupiter) radio continuum: solar system

\section{INTRODUCTION}

Ground-based radio astronomy observations of Jupiter, beginning nearly 40 years ago, established early the necessity of particle acceleration and nonthermal wave-particle interactions to explain both the decimetric and the decametric components of the Jovian emission (Carr \& Gulkis 1969). The decimetric emission, which has a peak flux near $1000 \mathrm{MHz}(\lambda=30$ $\mathrm{cm}$ ), is due to the synchrotron emission process powered by electrons accelerated up to tens to hundreds of $\mathrm{MeV}$ by radial diffusion from the outer magnetosphere into about $2 R_{\mathrm{J}}\left(R_{\mathrm{J}}=\right.$ Jovian radius, $71,000 \mathrm{~km}$ ). The inward flux of charged particles conserves the first two adiabatic invariants of motion while violating the third (Chang \& Davis 1962). The particle source is believed to be the solar wind, and there is some evidence that the decimetric emission waxes and wanes with solar wind variations with approximately a 2 year lag (Bolton et al. 1989). This synchrotron flux is extremely intense, permitting relatively easy detection of the emission from Earth and the generation of radio maps of the planet that manifest clear asymmetries in the planet's global magnetic field (e.g., de Pater $1981)$.

The decametric component (DAM) of the Jovian emission is only observed at frequencies below $39.5 \mathrm{MHz}$ and presents a highly complicated morphology as shown by the Voyager planetary radio astronomy (PRA) results (e.g., Warwick et al. 1979; Carr, Desch, \& Alexander 1983). The radiation is primarily in the extraordinary mode and is usually attributed to an electron cyclotron emission process. Hence the emission frequency is tied to the magnetic field strength at the point of emission through the equation $\nu=2.8 B$, where $\nu$ is the emission frequency in $\mathrm{MHz}$ and $B$ is the magnetic field strength in gauss. The observation of a $39.5 \mathrm{MHz}$ high-frequency cutoff in the DAM was early evidence that Jupiter's cloud top magnetic field strength must be near $14 \mathrm{G}$, a fact later confirmed by Pioneer 10 (Acuna \& Ness 1976; Smith, Davis, \& Jones 1976). The extraordinary radiated power of the decametric emission, upward of $10^{13} \mathrm{~W}$, is explained by a maser-induced radiation process known as the electron cyclotron maser ( $\mathrm{Wu} \&$ Lee 1979; Melrose \& Dulk 1982). An important feature of the DAM from the standpoint of particle acceleration processes is the fact that much of the emission is driven by Jupiter's innermost Galilean satellite, Io. Jupiter and Io constitute an electrodynamic engine (e.g., Hill, Dessler, \& Goertz 1983) that is capable of accelerating electrons to several or tens of keV, creating an electron beam that propagates along the magnetic flux tube connecting Io with the planet's cloud tops (Goldstein \& Goertz 1983). Along the way, the Io-accelerated electron beam generates DAM at frequencies down to (but not below) about $2 \mathrm{MHz}$ (Desch \& Carr 1978). The Jovian S-bursts, or millisecond bursts, with their persistent and even predictable negative frequency drifts, are a particularly dramatic manifestation of Io-controlled emission. Observations at high frequencies $(32 \mathrm{MHz}$ ) strongly suggest that the S-bursts are due to $\mathrm{keV}$ electrons that have been accelerated upward into the burst emission region from near the foot of the Io flux tube (Desch, Flagg, \& May 1978).

The synchrotron emission process is understood well enough that the information contained in decimeter-wave radio maps of Jupiter can be inverted to reveal some information about high-energy particle populations deep inside the Jovian magnetosphere. Conversely, the nature of the Io-Jupiter interaction and subsequent particle acceleration are poorly understood. Moreover, satellite and spacecraft observations have shown that the Jovian radio spectrum in fact extends down to the lowest frequencies at which radio emission can escape from the magnetosphere; this lower limit is set by the (time-variable) density of the planet's magnetosheath, generally corresponding to an escaping wave frequency of about $5 \mathrm{kHz}$. None of this very low frequency radiation, of which there are at least a half-dozen separate and distinct components (e.g., Stone et al. 1992b), is correlated with Io and hence requires delineation of alternative acceleration mechanisms that can provide the free energy necessary for substantial wave growth. 
In the present paper we will focus on two interesting radio and plasma wave components that have been observed in detail for the first time following the Ulysses spacecraft flyby of Jupiter in early 1992 (Kaiser et al. 1992; Stone et al. 1992b). In the case of both components, complementary experiments shed some light on the importance of particle acceleration in understanding the radio phenomena. The first component is the quasi-periodic (QP) emission, which covers a broad range of frequencies and resembles the S-bursts in some ways, although the QP bursts are not triggered by Io. High-energy particle beams have been observed in close association with the QP bursts; however, the timing of the two phenomena suggests that an intermediate agent is at work, perhaps related to a substorm-like process at Jupiter. The second component is Jovian whistler-mode auroral hiss. The generation mechanism and approximate particle energy responsible for the auroral hiss have been determined by an in situ magnetoplasma experiment performed on board Spacelab 2 (Farrell et al. 1988) in which a detector is flown across the path of a particle beam injected from the Space Shuttle. This illustrates the importance of controlled experiments in understanding space plasma phenomena.

\section{OBSERVATIONS}

Principal among the experimental results described in this paper are very recent data from the Ulysses spacecraft, which carries a complement of radio and plasma wave receivers ( the URAP experiment) designed to make observations from 0.08 $\mathrm{Hz}$ to $940 \mathrm{kHz}$ observing frequency (Stone et al. 1992a). We will also make use of earlier Voyager observations of the QP bursts from the plasma wave science (PWS) instrument (Scarf \& Gurnett 1977). The Shuttle Spacelab 2 mission experiment is described by Farrell et al. (1988), and the Ulysses cosmic and solar particle (COSPIN) investigation is described by Simpson et al. (1992a).

\subsection{Quasi-periodic Bursts}

Figure 1 (Plate 1) shows a frequency-time radio spectrogram of QP bursts observed by the Ulysses URAP experiment. At the time of the observations ( 1992 February 1) the spacecraft was $\sim 140 R_{3}$ sunward of the planet and inbound. The bursts are characterized by single-frequency durations on the order of a few minutes with total episodes lasting typically 1-2 hr (Kaiser et al. 1992). The burst frequency range extends from about $50 \mathrm{kHz}$ down to whatever intervening plasma frequency acts as a cutoff of the emission, usually about $5 \mathrm{kHz}$. The isotropic radiated power of the bursts is on the order of $3 \times 10^{7}$ $\mathrm{W}$ (compare with the $10^{13} \mathrm{~W}$ mentioned earlier for the Jovian DAM ). Perhaps most important, the bursts do not occur randomly in time but appear quasi-periodically with periods of 10-20 minutes. This is supported quantitatively in Figure 2, where we show a power spectrum of a several-hour-long episode of QP bursts in which the dominant spectral peak is at 15 minutes.

High-resolution Voyager PWS measurements (Kurth, Gurnett, \& Scarf 1989) provided the first evidence of QP bursts. At that time they were referred to as "Jovian type IIIs" because their frequency drift resembled that of solar type III bursts. An episode shown in Figure 3 illustrates their characteristic nega-

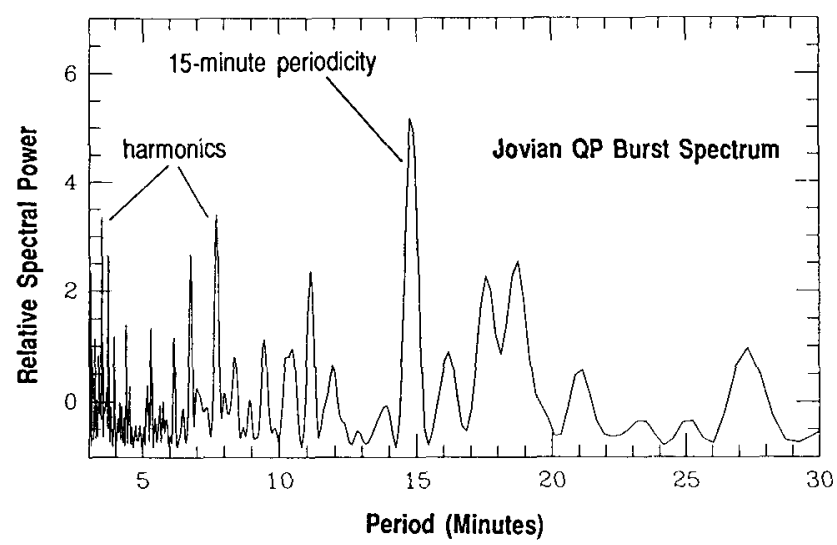

FIG. 2.-Power spectrum of an episode of $Q P$ bursts showing the dominance of power near 15 minute period. The higher frequency harmonics are probably spurious.

tive drift. This figure also shows how the bursts in a given 15 minute group can actually be made up of two or three subbursts separated by only a few minutes.

The name "QP bursts" is preferred to "Jovian type III," not only because it stresses the most important characteristic of the bursts, namely, their periodicity, but also because it can be shown that the shape of the QP burst is due merely to a propagation effect occurring in the Jovian magnetosheath after generation takes place. An illustration of this effect is shown in Figure 4. (Soon after Ulysses entered the Jovian magnetosphere the QP bursts disappeared, suggesting that they are in fact generated very close to the sheath and beamed outward.) As the wave propagates out of the magnetosphere to the spacecraft, it must transit the magnetosheath and must therefore suffer some degree of group velocity dispersion. The wave group velocity is given by

$$
v_{g}=c\left(1-\frac{\nu_{p}^{2}}{\nu^{2}}\right)^{1 / 2}
$$

where $\nu_{p}$ and $\nu$ are the electron plasma and wave frequencies, respectively. Since the QP bursts are observed down to frequencies comparable to the local plasma frequency in the sheath itself, the group velocity dispersion can be considerable at the lowest frequencies. Additionally, if the waves are incident on the sheath at an angle (Fig. 4), the lower frequencies will suffer more severe refraction and hence propagate longer in the sheath (assumed to be a curved slab), contributing to the observed drift. A straightforward calculation of the expected dispersion using equation ( 1 ) yields a model for the burst leading edge as a function of frequency. This is compared with observation in Figure 5. Here the QP burst in Figure 3 whose onset is at $1105 \mathrm{hr}$ spacecraft event time is greatly enlarged to show details of its curved leading edge. The agreement between the computed dispersion and burst leading edge is obvious. For this calculation, a sheath density of $0.85 \mathrm{~cm}^{-3}$ and ray path length through the sheath of $35 R_{\mathrm{J}}$ were used, consistent with typical in situ observations of the magnetosheath from Voyager (Lepping et al. 1981; Scudder, Sittler, \& Bridge 1981 ). It is assumed that all frequencies are generated simultaneously in the source region. The second-order effect of fre- 


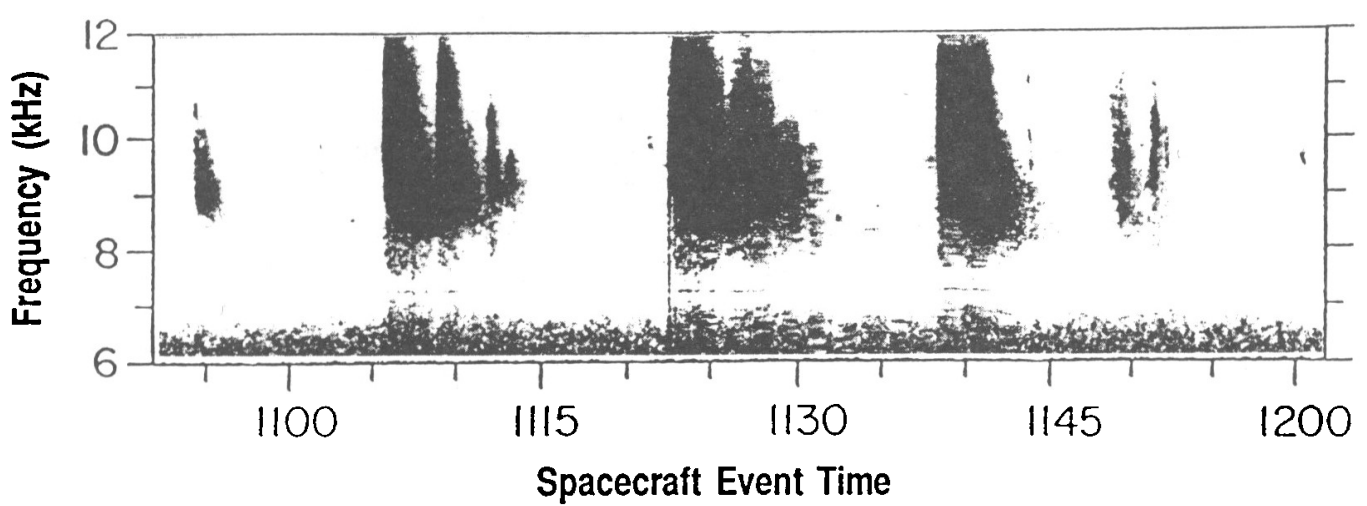

Fig. 3. - High-resolution spectrogram of Voyager 1 plasma wave science (PWS) data showing QP (Jovian type III) bursts on 1979 February 28 at a distance from Jupiter of $87.5 R_{\mathrm{J}}$. Spacecraft event time is in hours. (Figure adapted from Kurth et al. 1989.)

quency-dependent wave refraction was ignored in this calculation.

After the planetary flyby, when Ulysses was outbound, QP bursts were observed once again. Outbound the spacecraft was at negative jovigraphic latitudes (positive inbound) and in the evening local time sector (morning inbound). This change in perspective apparently resulted in the detection of a new type of QP burst because, as seen in Figure 6 (Plate 2) the QP bursts observed outbound now manifested a periodicity of 40-50 minutes, about 3 times the periodicity seen inbound. Further,

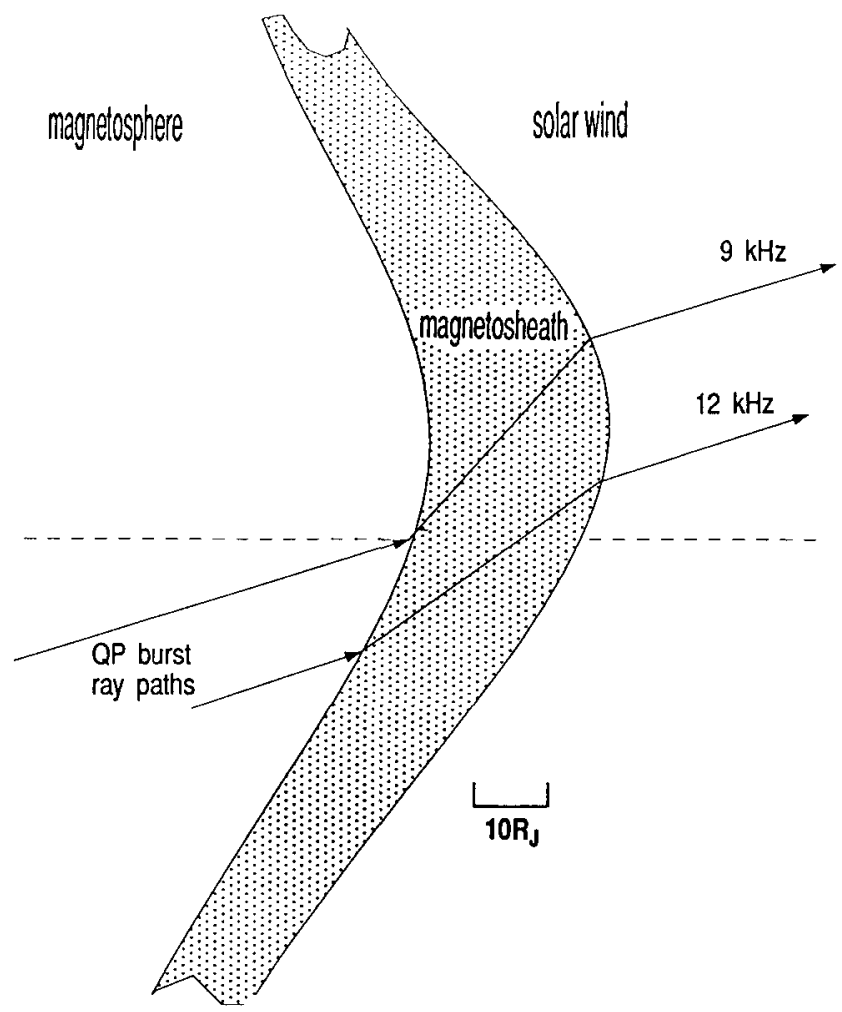

FiG. 4.-Cartoon depiction of the QP burst ray paths through the Jovian magnetosheath from a source region inside the magnetosphere. Dispersion of the rays and enhanced refraction at lower frequencies contribute to the observed negative drift of the burst leading edge. the frequency range was greater, extending to about $200 \mathrm{kHz}$ and the radiated power perhaps somewhat greater $\left(10^{8} \mathrm{~W}\right)$. The 15 minute QP bursts were also occasionally observed after encounter, so it seems that the 40 minute bursts are a separate, but somehow related, component.

Of particular relevance here is the fact that during at least one QP episode seen outbound, impulsive bursts of high-energy electrons $(E>8.9 \mathrm{MeV})$ with net flow away from the planet were observed by the COSPIN experiment on Ulysses (Simpson et al. 1992b). These electron bursts exhibited the same $\sim 40$ minute periodicity as the QP emission, but with somewhat longer decay times. (A similar phenomenon was observed by the Voyager 1 spacecraft in 1979 when bursts of field-aligned $0.5 \mathrm{MeV}$ protons with a periodicity of $\sim 40 \mathrm{~min}$ utes were detected on the outbound pass through Jupiter's predawn magnetosphere; Schardt, McDonald, \& Trainor 1981.)

The Ulysses electron bursts are compared with the radio burst observations in Figure 7 . The close association between

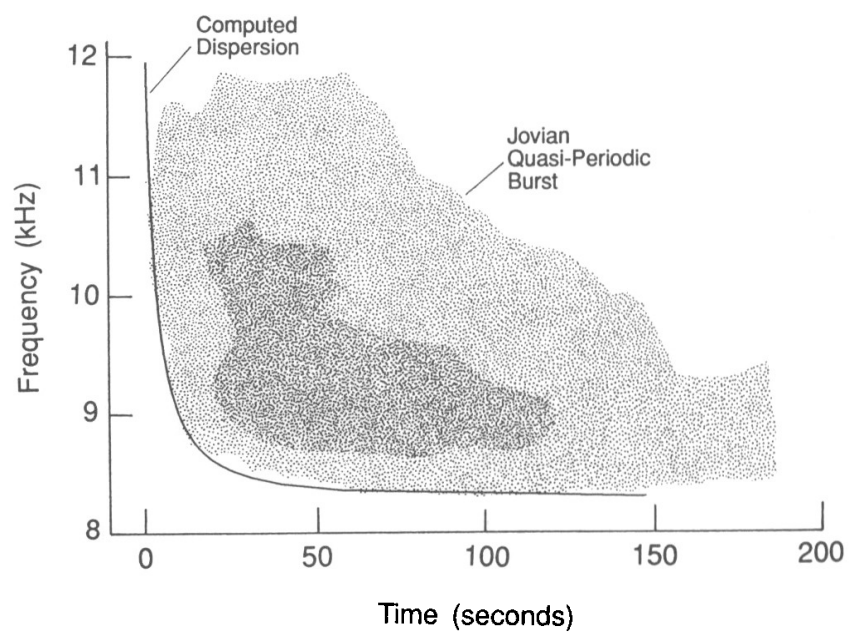

FIG. 5.-Comparison of the observed leading edge of a QP burst and the computed shape based on group velocity dispersion through the Jovian magnetosheath. The burst shown here is a greatly enlarged version of the QP burst shown in Fig. 3, whose onset is at 1105 hours spacecraft event time. The computed dispersion through the magnetosheath is the line that tracks along the leading edge of the burst. 


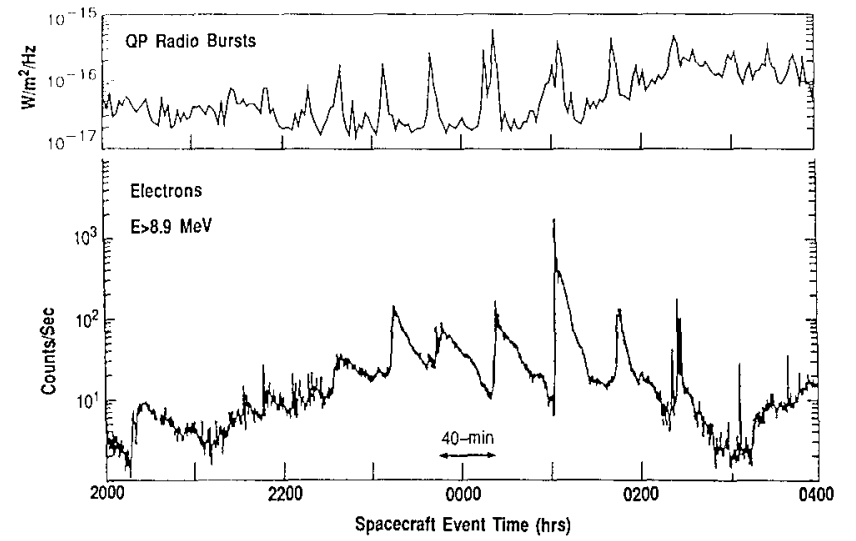

FIG. 7.-COSPIN electron data $(E>8.9 \mathrm{MeV})$ compared with the QP bursts detected by URAP. The spacecraft is outbound from Jupiter at a distance of $53 R_{\mathrm{J}}$ at $0 \mathrm{hr}$ spacecraft event time. (COSPIN data adapted from Simpson et al. 1992b.)

the two phenomena is evident. A superposed epoch analysis (Fig. 8) illustrates their phase relationship. With the assumption that each radio burst is associated with the nearest electron burst and with the electron burst onsets defined as the zero phase of the epoch, the phase of the radio bursts is such that their onsets occur, on average, about 8 minutes prior to the

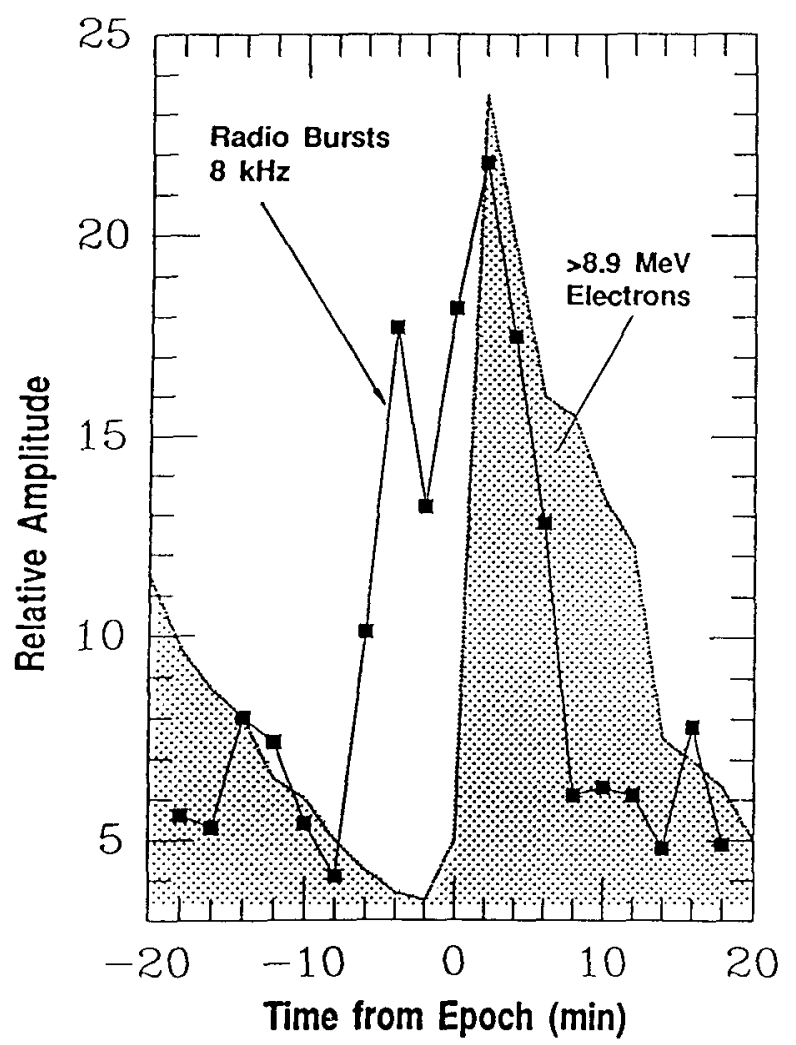

FIG. 8.-Superposed epoch (day 41-42, 1992) analysis results based on a superposition of the COSPIN energetic electron data with the QP bursts detected by URAP (see Fig. 7). Onset times of the electron bursts were defined as zero. The onsets of the QP bursts precede the electron bursts by about 8 minutes on average. arrival of the particle beam at the spacecraft. This observation presents something of a problem. Although the phenomena are clearly related in some way, the large temporal offset implies that since both the waves and the particles travel essentially at velocity $c$, there must be an intermediate step or steps between wave generation and particle acceleration. The particles themselves do not give rise to the radio emission.

We have investigated this further by noting that at the time of the observed correlation, Jupiter's magnetosphere was undergoing a large-scale compression due to a major enhancement in the solar wind density. The solar wind shock and density spike were observed about 16 days earlier by IMP 8 near the Earth (Lepping et al. 1992). At Jupiter the compression resulted in significant enhancements in Jupiter's radio emission output throughout the low-frequency bands. Simpson et al. (1992b) noted that the rapid onset of the electron bursts was suggestive of the explosive magnetic (tail) merging events seen at Mercury, but with the Jovian electron source directions implying an auroral source. It is highly possible, then, that an Earth-like magnetic substorm process is at work during the events described here and that the planet's magnetic tail is involved at least in the initial conversion of stored magnetic energy into free energy. In support of this, we note that there is evidence from Voyager observations made some years ago (Lepping 1986; Kurth et al. 1982) of a tearing away of the Jovian tail, probably caused by field line reconnection, during a solar wind sector boundary passage. The Voyager observations were made in the Jovian tail, far downstream of the planet. The Ulysses observations may be evidence of the same phenomenon but from a very different perspective.

\subsection{Auroral Hiss}

The second component of Jovian emission that provides some information on particle acceleration processes is the newly discovered auroral hiss emission. At Earth, observations of auroral hiss are sometimes taken as evidence of emission associated with a population of "inverted-V" electrons. These electrons, so-called for their appearance in electron spectrograms, are believed to be accelerated through a magnetic fieldaligned potential drop of several $\mathrm{keV}$ by means of an undefined auroral acceleration process, ultimately powered by the solar wind. The Ulysses observations of auroral hiss (Stone et al. 1992b) were the first evidence of this emission and first inference of this type of auroral acceleration process at Jupiter.

Figure 9 shows the observations from Ulysses of three consecutive episodes of auroral hiss. Both the wave electric (E) and magnetic ( B ) field data are shown in spectrogram format. The observed variations in the in situ electron plasma $\left(f_{p}\right)$, cyclotron $\left(f_{c}\right)$, and lower hybrid $\left(f_{\mathrm{lh}}\right)$ frequencies are superposed on the figure. The characteristic funnel-shaped structure, apparent primarily in the magnetic field data, and the restriction of the waves to frequencies below both $\left(f_{p}\right)$ and $\left(f_{c}\right)$ but above $\left(f_{\mathrm{lh}}\right)$, confirm the identification as whistler-mode hiss. Model ray tracing in which the source is located on auroral field lines illuminating the spacecraft when it is at high magnetic latitudes agrees with the observed start and stop times of the emission, confirming its source as the (northern) auroral region (Farrell et al. 1993).

Identification of the generation mechanism and electron energies responsible for the auroral hiss comes indirectly from a 

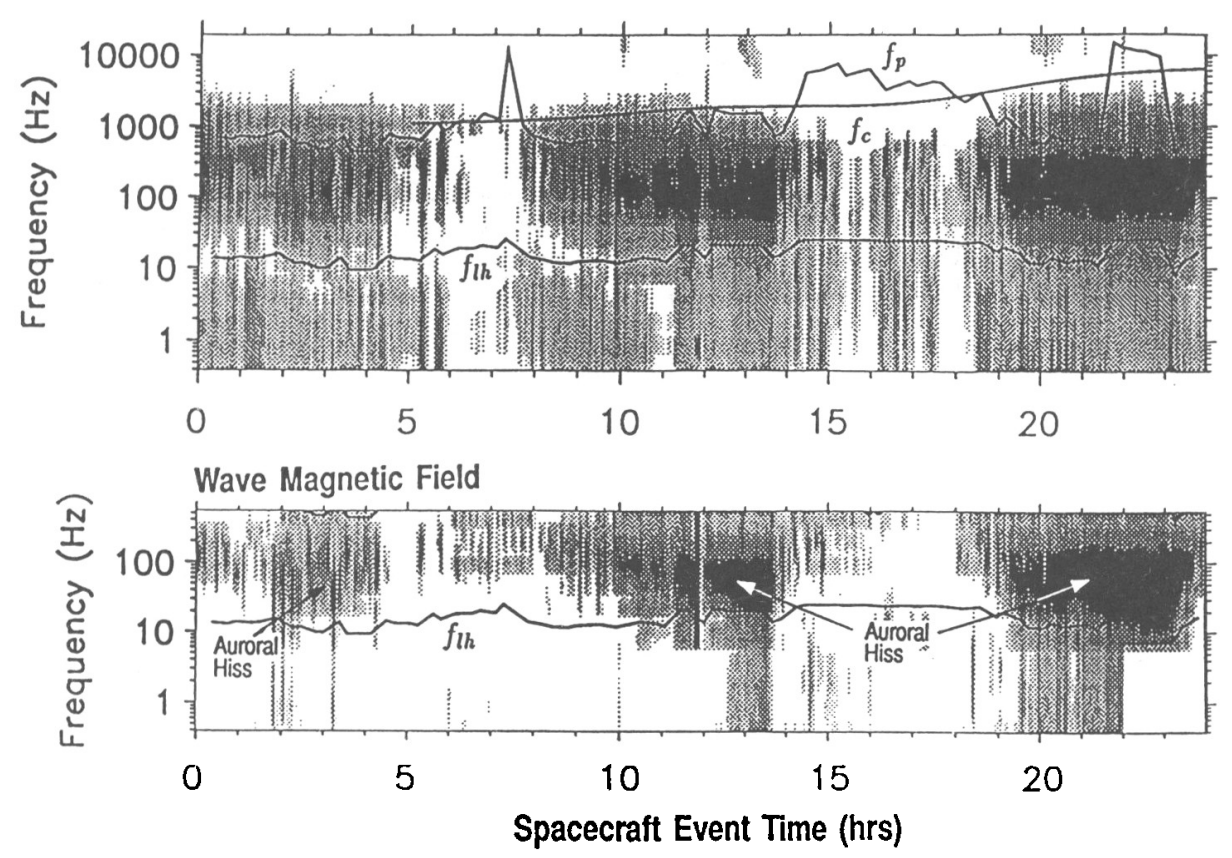

Fig. 9.-Frequency-time spectrogram from Ulysses of wave electric and magnetic field observations of Jovian auroral hiss. The local electron plasma, cyclotron, and lower hybrid frequencies $\left(f_{p}, f_{c}, f_{l h}\right)$ are superposed on the figure. (Figure adapted from Farrell et al. 1993.)

unique experiment performed from the Space Shuttle. A freeflying plasma wave detector (University of lowa PDP) was flown $200 \mathrm{~m}$ above the Shuttle and into the path of a beam of 1 $\mathrm{keV}$ electrons accelerated by the Stanford electron generator (Farrell et al. 1988). The characteristic whistler-mode funnelshaped emission was observed and identified as being due to a coherent Cerenkov radiation based on the measured emissivity. The measured $c \mathrm{~B} / \mathrm{E}$ ratios were consistent with emission that was weakly electromagnetic. In the Jovian case, the $c \mathbf{B} / \mathrm{E}$ ratios indicate that the auroral hiss is more strongly electromagnetic, consistent with electrons of somewhat higher energy than the $1 \mathrm{keV}$ particles observed from the Shuttle. Although the precise energies involved are not known, it seems likely that the $\sim 3 \mathrm{keV}$ energies seen at Earth in "inverted- $V$ " precipitation events would be capable of generating the auroral hiss at Jupiter.

\section{SUMMARY}

Despite their indirect link to particle acceleration processes, several Jovian radio components provide information on ener- getic charged particle populations in the Jovian magnetosphere. The synchrotron emission is caused by the inward radial diffusion and energization of particles to tens to hundreds of $\mathrm{MeV}$ via a process that may require several years to go from bow shock to inner magnetosphere. Jovian millisecond bursts seem to originate from cloud top electrons accelerated upward along the Io flux tube to energies of a few keV. Several lines of evidence suggest that the newly discovered quasi-periodic (QP) bursts, which are sometimes associated with fluxes of $E>9 \mathrm{MeV}$ electrons, may be evidence of a substorm-like process at Jupiter. Finally, the definitive observation of whistlermode auroral hiss at Jupiter, generated via a coherent Cerenkov process, suggests that $\mathrm{keV}$ electrons also populate the planet's auroral field lines.

I am greatly indebted to R. B. McKibben for making available the COSPIN electron data. Throughout this study I have benefited greatly from discussions with M. L. Kaiser, W. M. Farrell, and R. J. MacDowall.
Acuna, M. H., \& Ness, N. F. 1976, J. Geophys. Res., 81, 2917

Bolton, S. J., Gulkis, S., Klein, M. J., de Pater, I., Thompson, T. J., 1989, J. Geophys. Res., 94, 121

Carr, T. D., Desch, M. D., \& Alexander, J. K. 1983, in Physics of the Jovian Magnetosphere, ed. A. J. Dessler (Cambridge: Cambridge Univ. Press), 226

Carr, T. D., \& Gulkis, S. 1969, ARA\&A, 7, 577

Chang, D. B., \& Davis, L. 1962, ApJ, 136, 567

de Pater, I. 1981, A\&A, 93, 370

Desch, M. D., \& Carr, T. D. 1978, AJ, 83,828

Desch, M. D., Flagg, R. S., \& May, J. 1978, Nature, 272, 38

Farrell, W. M., Gurnett, D. A., Banks, P. M., Bush, R. I., \& Raitt, W. J. 1988, J. Geophys. Res., 93, 153

\section{REFERENCES}

Farrell, W. M., et al. 1993, J. Geophys. Res., submitted

Goldstein, M. L., \& Goertz, C. K. 1983, in Physics of the Jovian Magnetosphere, ed. A. J. Dessler (Cambridge: Cambridge Univ. Press), 317

Hill, T. W., Dessler, A. J., \& Goertz, C. K. 1983, in Physics of the Jovian Magnetosphere, ed. A. J. Dessler (Cambridge: Cambridge Univ. Press), 353

Kaiser, M. L., Desch, M. D., Farrell, W. M., MacDowall, R. J., Stone, R. G., Lecacheux, A., Pedersen, B.-M., \& Zarka, P. 1992, Geophys. Res. Lett., 19, 649

Kurth, W. S., Gurnett, D. A., \& Scarf, F. L. 1989, J. Geophys. Res., 94, 6917

Kurth, W. S., Sullivan, J. D., Gurnett, D. A., Scarf, F. L., Bridge, H. S., \& Sittler, E. C. 1982, J. Geophys. Res., 87, 10373 
Lepping, R. P. 1986, Adv. Space Res., 6, 269

Lepping, R. P., Burlaga, L. F., Klein, L. W., Jessen, J. M., \& Goodrich, C. C. 1981 , J. Geophys. Res., 86, 8141

Lepping, R. P., et al. 1992, IMP-8 Solar Wind Magnetic Field and Plasma Data in Support of Ulysses-Jupiter Encounter (NASA Internal Document, 12-25-92)

Melrose, D. B., \& Dulk, G. A. 1982, ApJ, 259, 844

Scarf, F. L., \& Gurnett, D. A. 1977, Space Sci. Rev., 21, 289

Schardt, A. W., McDonald, F. B., \& Trainor, J. H. 1981, J. Geophys. Res. 86,8413
Scudder, J. D., Sittler, E. C., \& Bridge, H. S. 1981, J. Geophys. Res., 86, 8157

Simpson, J. A., et al. 1992a, A\&AS, 92, 365 1992b, Science, 257, 1543

Smith, E. J., Davis, L., \& Jones, D. E. 1976, in Jupiter, ed. T. Gehrels (Tucson: Univ. Arizona Press), 788

Stone, R. G., et al. 1992a, A\&AS, 92, 291 1992b, Science, 257, 1524

Warwick, J. W., et al. 1979, Science, 204, 995

Wu, C. S., \& Lee, L. C. 1979, ApJ, 230, 621 